We report the case of a 13-year-old boy with severe obesity (BMI $>99^{\circ}$ p.le), polyuria and polydipsia. Physical examination showed abdominal adiposity, acanthosis nigricans (neck, limb folds and trunk) and no signs of Cushing syndrome. Laboratory examination showed fasting hyperglycaemia (293 mg/dl), HbA1c 12.8\%, negative autoantibody screening concerning type 1 diabetes mellitus and glycosuria without ketonuria. Fasting insulin and C-peptide values and after oral-glucose tolerance test were compatible with type 2 diabetes mellitus. Lipid state was normal (total cholesterol $147 \mathrm{mg} / \mathrm{dl}$, HDL-cholesterol $35 \mathrm{mg} / \mathrm{dl}$ and triglycerides $159 \mathrm{mg} / \mathrm{dl}$ ). Further specific examinations showed: left ventricle hypertrophy, borderline hypertension and hepatic statosis. The stabilization of glycemic values was achieved with a $500 \mathrm{mg}$ metformin-based therapy (three times daily), progressively increased up to $2000 \mathrm{mg}$, and a $2200 \mathrm{kcal}$ diet. As a result, the blood glucose values improved as well as the glycated $\mathrm{Hb}$ (reduced to $7.9 \%$ ) while the weight increased. After 2 years, due to the low compliance to the diet, the child was admitted within a multidisciplinary structure (pharmacologic therapy, aerobic fitness, nutritional program) where he stood three times, one month per time. The results were a weight decrease and an improvement of the glycolipidic metabolism.

However, back to home, the obesogenic context and the low-diet-compliance increased the child's weight up to a $60 \mathrm{BMI}$ and worsened the glycolipid profile, triggering a new admission in our department and, after the ethical committee approach, he started therapy with Exenatide $(10 \mathrm{mg} \times 2$ injections/d) in addition to the metformin. Two months later a weight and hungryattitude decrease was achieved $(-10 \mathrm{~kg})$.

\title{
26 - Single nucleotide polymorphisms of ADIPOQ gene and metabolic syndrome in European adolescents
}

\author{
E Erhardt ${ }^{1}$, S Bokor ${ }^{1}$, A Meirhaeghe ${ }^{2,3,4}$, RJ Ruiz ${ }^{5}$, K Widhalm $^{6}$, M Gonzalez-Gross ${ }^{7}$, \\ LA Moreno ${ }^{8}$, D Molnar ${ }^{1}$ and J Dallongeville ${ }^{2,3,4}$ on behalf of the HELENA \\ Study Group \\ 'Department of Paediatrics, University of Pecs, Pecs, Hungary: ${ }^{2}$ Institut Pasteur de Lille, Lille, France; \\ ${ }^{3}$ INSERM, U744, Lille, France: ${ }^{4}$ Université Nord de France, Lille, France: ${ }^{5}$ Unit for Preventive Nutrition, \\ Department of Biosciences and Nutrition at NOVUM, Karolinska Institutet, Stockholm, Sweden: \\ ${ }^{6}$ Department of Pediatrics, Medical University of Vienna, Vienna, Austria: ${ }^{7}$ Facultad de \\ Ciencias de la Actividad Fisica y del Deporte - INEF, Universidad Politecnica de Madrid, Madrid, \\ Spain: ${ }^{8}$ GENUD Research Group, Escuela Universitaria de Ciencias de la Salud, Universidad de \\ Zaragoza, Zaragoza, Spain
}

Introduction: Adiponectin may affect vascular function and mediate obesity-related vascular disorders including hypertension, diabetes mellitus and atherosclerosis. In the present study, we investigated the effect of polymorphisms (SNP) in the adiponectin $(A D I P O Q)$ gene on components of metabolic syndrome (MS) in European adolescents.

Method: Altogether fifteen SNP were genotyped by Illumina in the HELENA Study ( $n$ 1155, 12-17-year-old European adolescents). The studied phenotypes were BMI, waist circumference, blood pressure and plasma triglyceride, cholesterol and glucose levels.

Results: rs822393 (frequency: 0·21), rs7649121 (frequency: 0.15) and rs17366743 (frequency: 0.02) were associated with lower plasma HDL-cholesterol in adolescents $(P=0 \cdot 001, P=0 \cdot 00008$ and $P=0 \cdot 001$, respectively).
Two SNP (rs3821799, rs3774261) were associated to have higher risk of increased waist/hip $(\mathrm{W} / \mathrm{H})$ ratio $(P=0.003$ and $P=0.001$, respectively). The average number of risk factors of MS was significantly lower $(P<0 \cdot 003)$ in carriers of at least one minor allele of rs822396 compared with the children who were homozygous for the common allele.

Conclusions: Using a candidate gene approach, we were able to detect significant associations between SNP of the ADIPOQ and components of MS in adolescents. These data may highlight the role of these adipokine in MS, especially in adolescents.

Funding: The HELENA Study received funding from the European Union's Sixth RTD Framework Program (Contract FOOD-CT-2005-007034) and the Spanish Ministry of Education (EX-2007-1124). 